\title{
MARIE BESSON
}

\section{Rang moyen et agrégation de classements}

Revue française d'automatique, d'informatique et de recherche opérationnelle. Recherche opérationnelle, tome 9, $\mathrm{n}^{\circ} \mathrm{V} 1$ (1975), p. 37-58.

<http://www.numdam.org/item?id=RO_1975_9_1_37_0>

(C) AFCET, 1975, tous droits réservés.

L'accès aux archives de la revue « Revue française d'automatique, d'informatique et de recherche opérationnelle. Recherche opérationnelle » implique l'accord avec les conditions générales d'utilisation (http://www.numdam.org/ legal.php). Toute utilisation commerciale ou impression systématique est constitutive d'une infraction pénale. Toute copie ou impression de ce fichier doit contenir la présente mention de copyright.

\section{Numdam}

Article numérisé dans le cadre du programme

Numérisation de documents anciens mathématiques

http://www.numdam.org/ 


\title{
RANG MOYEN \\ ET AGREGATION DE CLASSEMIENTS (*)
}

par Marie Besson $\left({ }^{1}\right)$

\begin{abstract}
Résumé. - La notion de rang est bien connue pour résumer une structure d'ordre total dans le cas d'ensembles finis.

Elle est généralisée ici aux structures de préordres quelconques, pour l'appliquer ensuite à certains problemes statistiques:

- obtention d'un préordre total à partir d'un ou plusieurs préordres quelconques,

- définition de distances entre éléments préordonnés (dans le but d'effectuer des typologies, par exemple).
\end{abstract}

\section{INTRODUCTION}

Pour les ensembles finis munis d'un ordre total, la notion de rang est bien connue. Nous nous proposons dans cet article de la généraliser au cas des préordres quelconques, et de l'appliquer ainsi à certains problèmes d'analyse multicritère, et notamment à l'agrégation de classements.

Considérons les préordres totaux, la détermination d'un rang n'est pas absolument évidente.

Soit par exemple une classe de 24 élèves ou 23 d'entre eux ont pour note $B$ et, le dernier a pour note $C$.

Devrons-nous dire, comme il est classique de le faire, que les 23 ex-acquo sont premiers (ont pour rang : 1) et que le dernier a pour rang 24 ?

Cela revient à donner une différence de rang égale à $24-1=23$.

Alors que si nous inversions notre point de vue à partir de la même situation, en considérant que le premier est celui qui a la plus mauvaise note, nous trouverions :

- un premier ayant pour rang 1

- 23 derniers ayant pour rang 2 .

(*) Reçu le 26 novembre 1973.

(1) I.U.T. Transport et Logistique, Paris XIII, Villetaneuse.

Revue Française d'Automatique, Informatique et Recherche Opérationnelle nº janv. 1975, V-1. 
Nous allons essayer de définir un rang plus "neutre», qui ne soit pas ainsi lié au sens par lequel on considère un même problème $\left({ }^{1}\right)$. Un rang correct sera tel que la différence de rangs, dans l'exemple que l'on vient de voir, ne soit ni « 1 », ni « 23 », mais la moyenne entre ces deux valeurs, c'est-àdire : 12 .

Nous lui donnerons le nom de rang moyen.

Après avoir défini un tel rang, nous étudierons ses diverses propriétés. Puis nous examinerons certaines de ses utilisations possibles dans le domaine de l'analyse multicritère. Ce sera essentiellement la façon d'obtenir un préordre total unique, à partir d'un ou plusieurs préordres quelconques (ce sera aussi une façon d'obtenir une distance entre des éléments préordonnés).

Nous présenterons ainsi une dérivée de la méthode Électre classique : l'utilisation du rang moyen permettra d'assouplir la demande d'information initiale, et de compléter l'information obtenue comme résultat.

\section{RANG MOYEN}

\subsection{Définition du rang moyen}

Soit $E$ un ensemble quelconque à $n$ éléments.

Soit $P$ un préordre quelconque sur $E$.

Soit $x$ un élément quelconque de $E$.

Soit $X^{\prime}(x)$ l'ensemble des éléments $y$ de $E$ vérifiant les relations

$y P x$ et $x \not P y$ (c'est l'ensemble des éléments strictement supérieurs à $x$, parfois noté Maj $(x)$ )

Soit $X^{\prime \prime}(x)$ l'ensemble des éléments $y$ de $E$ définis par les relations

$x P y$ et $y \not p x$ (c'est l'ensemble des éléments strictement inférieurs à $x$, parfois noté $\operatorname{Min}(x)$ )

( $X^{\prime}$ et $X^{\prime \prime}$ sont des applications de $E$ dans $P(E)$ ).

Considérons les trois applications $f, g$ et $r$ de $E$ dans $R^{+}$(ensemble des réels positifs) définies par les relations I, II, et III ci-dessous :

Rang $f$ :

$$
\text { I } \mid \begin{array}{ll}
f(x)=1 & \text { si } X^{\prime}(x)=\varnothing \\
f(x)=\operatorname{Sup}_{y \in X^{\prime}(x)} f(y)+\mid f^{-1} \operatorname{Sup}_{y \in X^{\prime}(x)}(f(y) \mid & \text { si } X^{\prime}(x) \neq \varnothing
\end{array}
$$

(1) Situation correspondant à une information se résumant à un préordre. 
c'est-à-dire $f(x)$ vaut 1 si aucun élément n'est strictement supérieur à $x$ d'après le préordre $P$; sinon $f(x)$ est égal à la plus grande valeur prise par $f$ dans $X^{\prime}$ augmentée du nombre d'éléments de $E$ ayant cette dernière valeur.

\section{Rang $g$ :}

$$
\text { II } \mid \begin{array}{ll}
g(x)=1 & \text { si } X^{\prime \prime}(x)=\varnothing \\
g(x)=\operatorname{Sup}_{y \in X^{\prime \prime}(x)} g(y)+\left|g^{-1} \operatorname{Sup}_{y \in X^{\prime \prime}(x)} g(y)\right| & \text { si } X^{\prime}(x) \neq \varnothing
\end{array}
$$

C'est-à-dire : $g(x)$ vaut 1 si aucun élément n'est strictement inférieur à $x$ d'après le préordre $P$; sinon, $g(x)$ est égal à la plus grande valeur prise par $g$ dans $X^{\prime \prime}$ augmentée du nombre d'éléments de $E$ ayant cette dernière valeur.

Rang $r$ :

$$
\text { III } \mid r(x)=\frac{f(x)-g(x)+n+1}{2}
$$

Nous conviendrons d'appeler « rangs" $\left({ }^{1}\right)$, ces trois applications $f, g$ et $r$; $r(x)$ étant le « rang moyen" de $x$ relatif au préordre $P$,

$f(x)$ étant le " rang descendant "

$g(x)$ étant le « rang ascendant ».

\subsection{Exemple de détermination des rangs associés à un préordre $P$ sur $E$}

Soit $E=\left\{x_{1}, x_{2}, x_{3}, x_{4}, x_{5}, x_{6}, x_{7}, x_{8}\right\}$.

Soit $P$ un préordre sur $E$, défini par sa décomposition en une relation d'équivalence $R$ et une relation d'ordre $T$ sur les classes formées par $R\left({ }^{2}\right)$.

On suppose $R$ définie elle-même par la donnée des classes d'équivalence correspondantes : $A, B, F, D$ et $E$.

$$
\begin{aligned}
& A=\left\{x_{1}, x_{2}\right\} \\
& B=\left\{x_{3}\right\} \\
& F=\left\{x_{4}, x_{5}, x_{6}\right\} \\
& D=\left\{x_{7}\right\} \\
& E=\left\{x_{8}\right\}
\end{aligned}
$$

$T$ est défini par les relations : $A T B, B T F, A T F, D T E$.

(1) A ne pas confondre avec les "fonctions de rang" ou "graduation »; voir annexe.

(2) Un préordre est équivalent à une relation d'équivalence dont les classes sont ordonnées.

$\mathrm{n}^{\circ}$ janvier $1975, \mathrm{~V}-1$. 
Si nous convenons de représenter une relation du type « $X T Y$ » par une flèche orientée de $X$ vers $Y$, nous aurons :

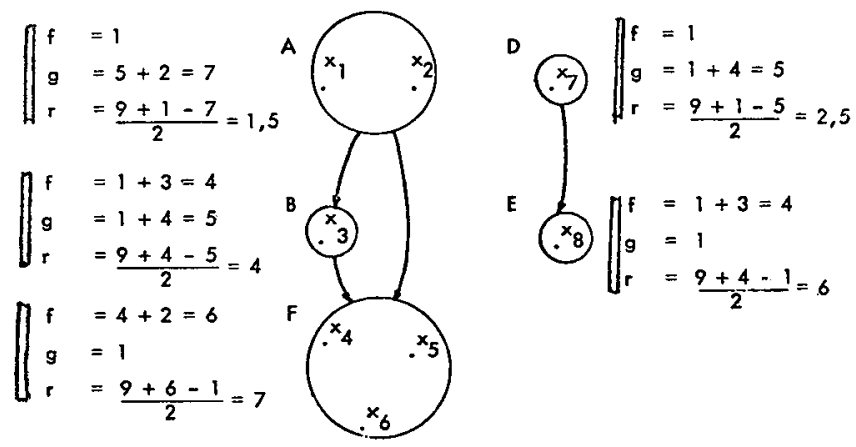

Figure 1

\subsection{Si $P$ est un préordre total}

Supposons $P$ défini par sa décomposition en classes d'équivalence ordonnées (totalement) $A_{1}, A_{2} \ldots A m$

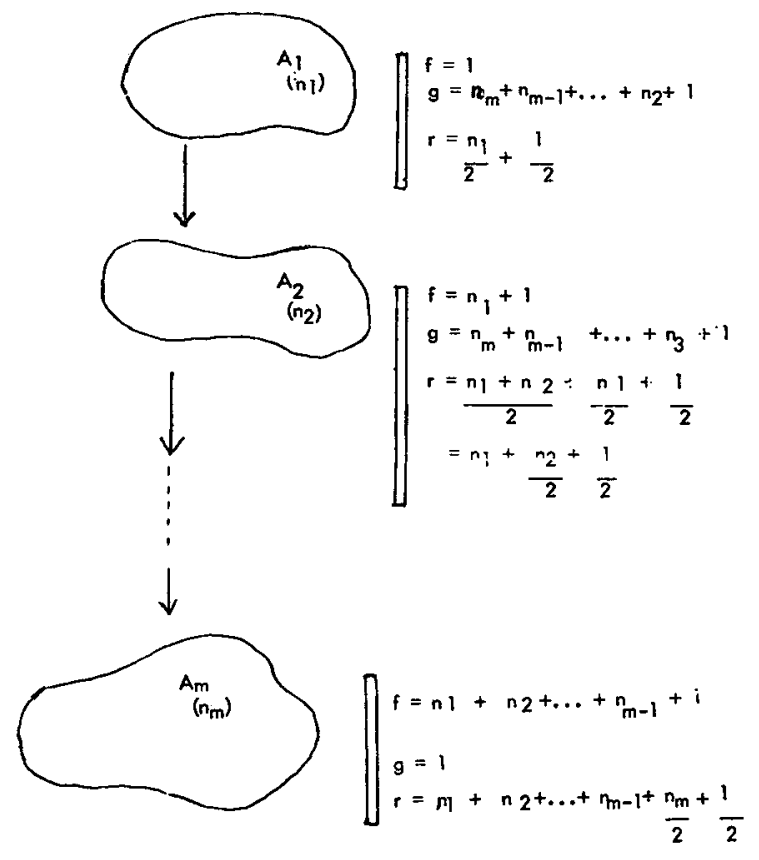

Figure 2

Revue Française d'Automatique, Informatique et Recherche Opérationnelle 
Plus généralement :

Soit $n_{i}$ le cardinal de $A_{i}$.

Soit $x \in A_{i}$

$$
\begin{aligned}
f(x) & =1+n_{1}+n_{2}+\ldots+n_{i-1} \\
g(x) & =1+n_{m}+n_{m-1}+\ldots+n_{i+1} \\
r(x) & =\frac{n+1+f(x)-g(x)}{2} \\
& =\frac{2\left(\sum_{j=1}^{i-1} n_{j}\right)+1+n_{i}}{2}=\sum_{1=1}^{i-1} n_{j}+\frac{n_{i}+1}{2}
\end{aligned}
$$

Remarque : Pourquoi ce nom " rang moyen "? Si chaque classe devient totalement ordonnée, la moyenne des rangs que l'on y trouve n'est autre que le rang moyen.

\subsection{Ordre total}

Si $P$ est un ordre total, nous aurons alors :

$(\forall x \in E)(r(x)=f(x)=($ nombre d'éléments de $E$ « meilleurs » que $x)+1)$

D'autre part :

$(\forall x \in E)(g(x)=n+1-f(x)=$ (nombre d'éléments de $E$ « moins bons » que $x)+1$ )

En effet, nous aurons alors pour tout $x$ de $E$.

$$
\begin{aligned}
\left|X^{\prime}(x)\right|+\left|X^{\prime \prime}(x)\right|+1 & =n \\
f(x) & =\left|X^{\prime}(x)\right|+1 \\
g(x) & =\left|X^{\prime \prime}(x)\right|+1
\end{aligned}
$$

Donc :

et :

$$
\begin{aligned}
f(x)-1+g(x)-1+1 & =n \\
g(x) & =n+1-f(x)
\end{aligned}
$$

D'autre part, nous aurons :

$$
\begin{aligned}
r(x) & =\frac{f(x)-g(x)+n+1}{2} \\
& =\frac{f(x)-n-1+f(x)+n+1}{2} \\
& =f(x)
\end{aligned}
$$

$\mathrm{n}^{\circ}$ janvier 1975, V-1. 
N.B.

La fonction $g$ est alors une graduation $\left({ }^{1}\right)$.

\subsection{Autres types d'ordres}

Nous nous proposons ici de calculer nos rangs dans des exemples très divers :

\subsubsection{Ordre quelconque}

$$
E=\{A, B, C, D, E, F, G, H, I\}
$$

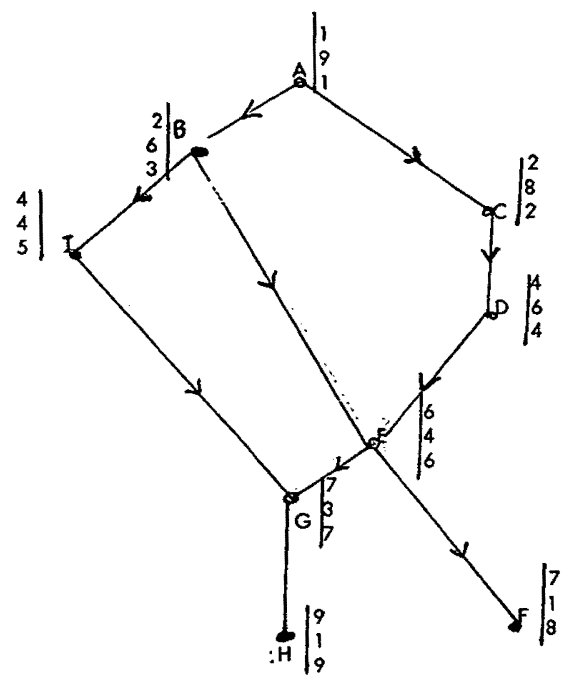

Figure 3

REMARQue : Une flèche de $X$ vers $Y$ indique que " $X P Y$ », $P$ étant la relation d'ordre considérée.

A côté de chaque élément de $E$, on trouve les valeurs des 3 rangs les unes sous les autres:

$$
\mid \begin{aligned}
& f \\
& g \\
& r
\end{aligned}
$$

(1) Voir annexe. 
Si nous rangeons ensuite les éléments de $E$, selon le rang moyen $r$, nous obtenons l'ordre total suivant :

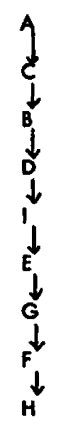

Figure 4

\subsubsection{Ordres gradués}

Certains ordres sont dits " gradués " $\left({ }^{1}\right)$. C'est le cas par exemple de ceux qui admettent un minorant universel et vérifient la condition de Jordan Dedekind $\left({ }^{1}\right)$. En voici un exemple.

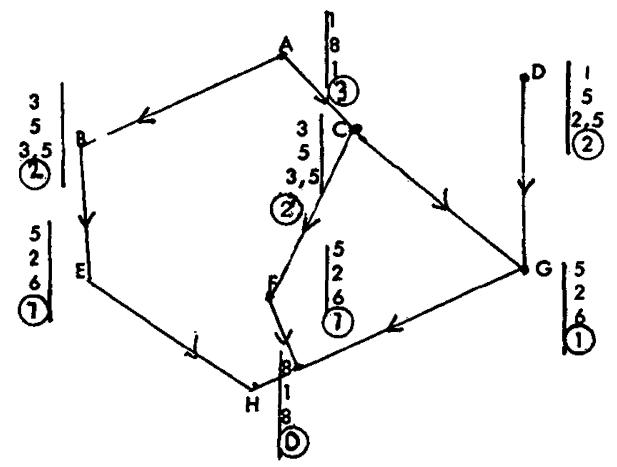

Figure 5

Nous avons pris ici comme graduation (valeur entourée) la hauteur de chaque élément.

REMARQUE :

Si dans un ensemble ordonné et gradué deux éléments ont même graduation, ils ont même rang ascendant $g$.

(1) Voir annexe.

$\mathrm{n}^{\circ}$ janvier 1975, V-1. 
Rangements comparés suivant

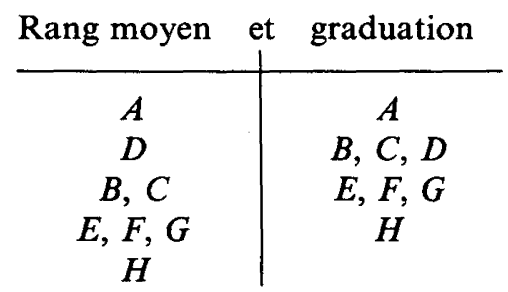

2.5.3. Treillis gradué $\left({ }^{1}\right)$

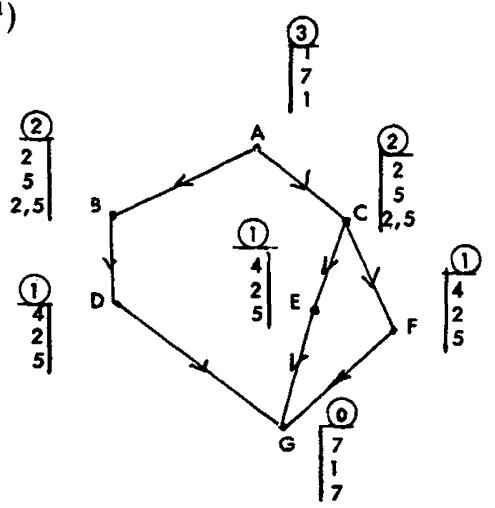

Figure 6

Nous avons pris comme graduation (valeur entourée), la hauteur de chaque élément, $h\left({ }^{1}\right)$.

\section{REMARQue :}

Si dans un treillis gradué, deux éléments ont même graduation, ils ont aussi même rang ascendant, même rang descendant et donc même rang moyen.

Les rangements comparés suivant $r, f, g$ ou $h$ sont donc identiques.

2.5.4. Treillis modulaire $\left({ }^{1}\right)$

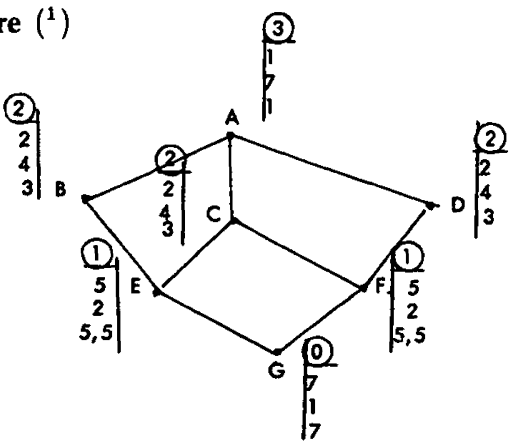

Figure 7

(1) Voir annexe. 
La graduation de chaque élément (hauteur) est comme précédemment, entourée sur le dessin.

Comme pour les treillis gradués, les rangements suivant $r, f, g$ ou $h$ demeurent identiques.

\subsection{Relation d'équivalence}

La notion de rang perd dans ce préordre particulier l'essentiel de son intérêt.

Il est triviạl de constater que nous avons alors, pour tout élément $x$ de $E$ :

$$
\begin{aligned}
f(x) & =1 \\
g(x) & =1 \\
r(x) & =\frac{f-g+n+1}{2}=\frac{n+1}{2}
\end{aligned}
$$

Tous les éléments de $E$ ont même rang moyen : la moyenne des rangs obtenus dans le cas d'un ordre total. Cela semble logique mais sans guère d'intérêt.

\section{UTILISATIONS}

\subsection{Généralités}

Nous venons ainsi de montrer comment, à partir d'un préordre quelconque dans un ensemble $E$ fini, nous pouvons obtenir une application de $E$ dans $R$, appelée « rang moyen », résumé de l'information initiale.

Observons les qualités de ce rang moyen :

1. Universalité. Unicité

Ce rang moyen s'applique à tout préordre et dans chaque cas, il est unique.

\section{Compatibilité}

Dans le cas particulier des ordres totaux, nous retrouvons le "rang", au sens trivial du terme, dans la langue française.

\section{Neutralité}

Certains rangs sont liés au sens par lequel on itère pour les déterminer.

C'est le cas des rangs ascendants et descendants. Le rang moyen au contraire est neutre par rapport au sens d'itération. 


\section{Sans à priori}

$\mathrm{Si}$ aucune information de rangement n'est donnée au départ (relation d'équivalence), aucune information ne sera fournie par notre rang moyen : pour chacun des éléments de $E$, il prendra en effet la même valeur : la moyenne de tous les rangs possibles.

\section{Correspondance entre préordre et rang moyen :}

Observons maintenant l'application qui fait correspondre un rang moyen à tout préordre quelconque.

Cette application $\alpha$ n'est pas injective. En effet, reprenons l'exemple du paragraphe (2.5.1). Il est clair que l'ordre

$$
\text { " } A C B D I E G F H \text { " }
$$

donnerait le même rang moyen :

$$
\begin{aligned}
& r(A)=1 \\
& r(C)=2 \\
& r(B)=3 \\
& r(D)=4 \\
& r(I)=5 \\
& r(E)=6 \\
& r(G)=7 \\
& r(F)=8 \\
& r(H)=9
\end{aligned}
$$

Par contre, la restriction $\alpha_{c}$ de cette application aux préordres totaux est injective (la démonstration est triviale).

Mais, ni $\alpha$, ni $\alpha_{c}$ ne sont surjectives évidemment.

Étudions maintenant certaines utilisations possibles.

\subsection{Passage d'un préordre quelconque à un préordre total}

Soit $P_{i}$ un préordre quelconque sur $E$.

Soit $r$ le rang moyen correspondant.

Le classement des éléments de $E$, selon ce rang moyen, donne un préordre total sur $E$. L'application correspondante (d'un préordre quelconque à un préordre total) est idempotente.

3.3. Passage des préordres quelconques pondérés à un préordre total unique

Soient $P_{1}, P_{2} \ldots P_{p}$ un ensemble de $p$ préordres quelconques sur $E$.

Soient $\alpha_{1}, \alpha_{2} \ldots \alpha_{p}$ des coefficients de pondération associés à ces préordres. 
Soient $x_{1}, x_{2} \ldots x_{n}$ les éléments de $E$.

Nous pouvons transcrire tous nos rangs moyens sur le tableau suivant :

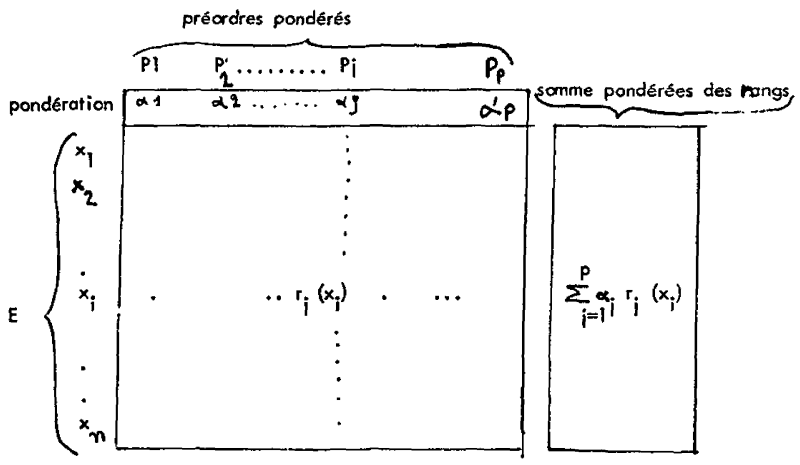

Figure 8

Nous calculons ensuite dans la dernière colonne la somme pondérée des rangs ainsi obtenus. Un classement final suivant cette somme pondérée nous donne un préordre total sur $E$, résumé de notre $p$-uplet de préordres quelconques et pondérés du départ.

Nous aurions pu également utiliser pour cela par exemple une dérivée de la méthode Electre classique décrite dans la partie IV de ce texte.

\subsection{Distance entre éléments préordonnés}

Une troisième utilité de ce rang moyen nous semble être la possibilité de déduire d'un $p$-uplet de préordres quelconques sur $E$, des distances entre les éléments de $E$.

Reprenons les mêmes notations que précédemment.

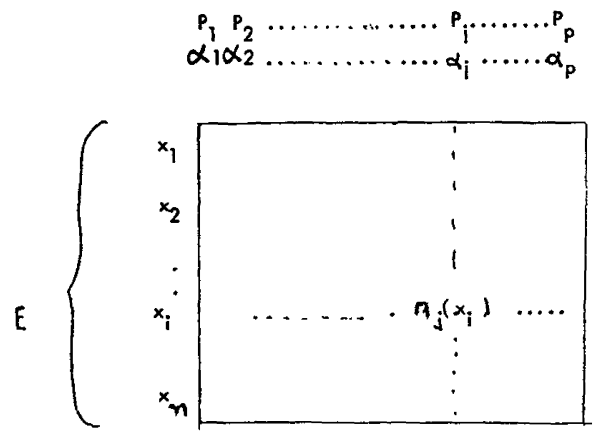

Figure 9

$\mathrm{n}^{\circ}$ janvier $1975, \mathrm{~V}-1$. 
Chaque élément $x_{i}$ de $E$ correspond ainsi à un vecteur de $\mathbf{R}^{p}$.

Toute distance sur $\mathbf{R}^{p}$ définit donc une distance sur $E$.

\section{REMARQUe :}

La détermination de distances entre éléments préordonnés d'un certain ensemble $E$, nous permettra notamment d'établir des typologies sur $E$ (voir [4] et [10]).

\section{UNE DERIVEE DE LA METHODE ELECTRE}

\subsection{Données}

Soit $E$ un ensemble de $n$ éléments $x_{1}, x_{2} \ldots x_{j} \ldots x_{n}$.

On suppose connu sur $E p$ préordres d'importances relatives $\alpha_{1}, \alpha_{2}, \ldots \alpha_{i}, \ldots \alpha_{p}$

$$
\text { Posons : } \quad \pi=\sum_{i \leqslant p} \alpha_{i}
$$

(on aura souvent : « $\pi=1 »$ ).

Le problème consiste à trouver un préordre $P$ sur $E$, résumant ces $m$ préordres pondérés.

\subsection{Ensembles de concordance}

Considérons $E x E$, ensemble des couples d'éléments de $E$.

Nous définirons une famille $Q$ de parties de $E x E$ de la manière suivante : avec

$$
\begin{gathered}
Q=[Q k / k \in(O, 1)] \\
(\forall(x, y) \in E x E)\left((x, y) \in Q k \Leftrightarrow \sum_{x P y} \alpha_{i} \geqslant k \cdot \pi\right)
\end{gathered}
$$

Nous conviendrons d'appeler $Q k$ « ensemble de concordance au niveau $k$ ». $Q k$ comporte donc tous les couples $(x, y)$ tels que la relation $x P y$ soit acceptée par un ensemble de préordres dont la somme des pondérations est supérieure ou égale à $k . \pi$.

Notons que :

$$
\left(k>k^{\prime}\right) \Rightarrow\left(Q_{k} \subset Q_{k^{\prime}}\right)
$$

C'est-à-dire il y a monotonie décroissante des $Q_{k}$ par rapport aux $k$.

$Q$ est une famille de parties incluses les unes dans les autres. 


\subsection{Ensembles de discordance}

Nous définirons ensuite une famille $D$ de parties de $E x E$ de la manière suivante :

$$
D=\left\{D_{j} / j \in(O, 1)\right\}
$$

avec $(\forall(x, y) \in E x E) \quad\left((x, y) \in D_{j} \Leftrightarrow \operatorname{Sup}_{P_{1}, P_{2} \cdots P_{p}}(\operatorname{Rang} y-\operatorname{Rang} x) \geqslant j(n-1)\right)$ (Il s'agit ici de rangs moyens.)

Nous conviendrons d'appeler $D_{j}$ « ensemble de discordance au niveau $j$ ».

$D_{j}$ comporte donc tous les couples $(x, y)$ d'éléments de $E$ tels que l'un au moins des critères $P_{1}, P_{2} \ldots P_{p}$ refuse l'inégalité « $x$ meilleur que $y$ », en plaçant $y$ au moins $j(n-1)$ rangs au-dessus de $x$.

Notons que :

$$
\left(j>j^{\prime}\right) \Rightarrow\left(D_{j} \subset D_{j^{\prime}}\right)
$$

donc $\left(j>j^{\prime}\right) \Rightarrow\left(C D_{j} \supset C D_{j^{\prime}}\right)$.

$(C$ signifie « complémentaire dans $E x E »)$.

\subsection{Relation binaire}

Considérons alors l'intersection de $Q k$ et du complémentaire de $D_{j}$ et enlevons-lui la diagonale de $E x E$ :

$$
I_{k j}=\left[Q k \cap C D_{j}\right]-[\text { Diagonale de } E x E]
$$

La diagonale en question est l'ensemble des couples $(x, y)$ tels que $x=y$. $I k j$ est une fonction croissante de $j$ et décroissante de $k$.

Nous conviendrons d'accepter la relation " $x$ meilleur que $y$ " si et seulement si : $(x, y) \in I_{k j}$.

\subsection{Fermeture réflexo-transitive}

Nous prendrons ensuite la fermeture réflexo-transitive de $I_{k j}$ dans le produit cartésien $E x E$ et nous appliquerons le rang moyen $r$ correspondant à ce préordre quelconque.

Le résultat de la méthode Electre n'est autre que le rangement des éléments de $E$ suivant $r$.

\subsection{Exemple d'application du programme Electre utilisé ici}

* 4 éléments dans l'ensemble de base

* 5 critères.

$\mathrm{n}^{\circ}$ janvier $1975, \mathrm{~V}-1$. 
Soit $E$ un ensemble quelconque à 4 éléments $(n=4)$

$$
E=\left\{x_{1}, x_{2}, x_{3}, x_{4}\right\}
$$

Supposons connus 5 préordres totaux sur $E: P_{1}, P_{2}, P_{3}, P_{4}$ et $P_{5}$ de même importance (équipondération : $(\forall i \leqslant 5)\left(\alpha_{i}=1\right)$ ).

Nous convenons de représenter chaque préordre $P_{i}$ par une colonne telle que :

$x_{j} P_{i} x_{k} \Leftrightarrow x_{j}$ se trouve, dans la colonne $i$, sur une ligne précédente, ou à la même ligne que $x_{k}$.

\begin{tabular}{|c|l|l|l|l|}
\hline$p_{1}$ & $p_{2}$ & $p_{3}$ & $p_{4}$ & $p_{5}$ \\
\hline$x_{1}$ & $x_{1} x_{2}$ & $x_{3}$ & $x_{2}$ & $x_{1}$ \\
$x_{3}$ & $x_{4}$ & $x_{1}$ & $x_{1} x_{3} x_{4}$ & $x_{2}$ \\
$x_{4}$ & $x_{3}$ & $x_{2}$ & & $x_{3}$ \\
$x_{2}$ & & $x_{4}$ & & $x_{4}$ \\
\hline
\end{tabular}

Figure 10

Tableau des rangs moyens correspondant

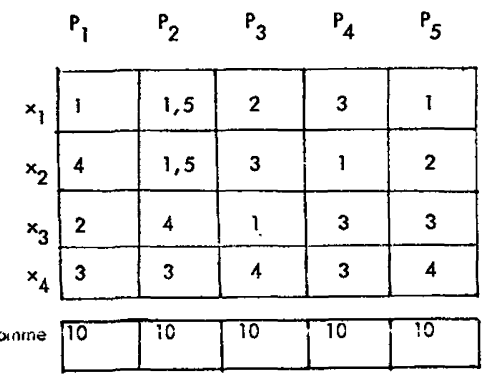

Exemple de calcul (pour $\left.P_{2}\right)$ :

$$
\begin{aligned}
f\left(x_{2}\right) & =1 \\
g\left(x_{2}\right) & =3 \\
z\left(x_{2}\right) & =\frac{n+1+f-g}{2} \\
& =\frac{5+1-3}{2}=1,5
\end{aligned}
$$

Figure 11

Tableau des concordances:

$$
q\left(x_{j}, x_{k}\right)=\sum_{i / x_{j} P_{i} x_{k}} q_{i}
$$

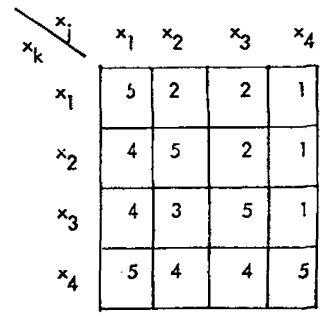

Figure 12 
Tableau des discordances

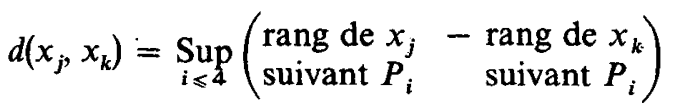

Niveau de concordance choisi : $80 \%$.

Niveau de discordance choisi : $40 \%$

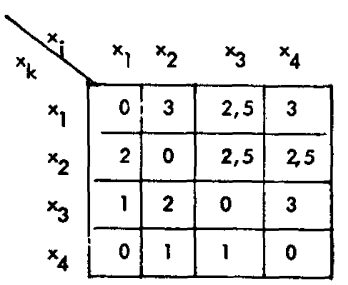

Figure 13

Donc : $80 \% \times$ somme des pondérations $=\frac{80}{100} \cdot 5=4$ et : $40 \%$ (nombre d'éléments de $E-1$ ) $=40 \% 4=1,6$.

Ensemble de concordance correspondant (couples d'éléments de $E$ dont la concordance est $\geqslant 4)$.

$$
\begin{array}{r}
Q_{(80 \%)}=\left\{\left(x_{1}, x_{1}\right),\left(x_{2}, x_{2}\right),\left(x_{3}, x_{3}\right),\left(x_{4}, x_{4}\right),\left(x_{1}, x_{2}\right),\left(x_{1}, x_{3}\right),\right. \\
\left.\left(x_{1}, x_{4}\right),\left(x_{2}, x_{4}\right),\left(x_{3}, x_{4}\right)\right\}
\end{array}
$$

Ensemble de discordance correspondant 'couples d'éléments de $E$ dont la discordance est $\geqslant 1,6)$

$D_{(40 \%)}=\left\{\left(x_{1}, x_{2}\right),\left(x_{2}, x_{1}\right),\left(x_{2}, x_{3}\right),\left(x_{3}, x_{1}\right),\left(x_{3}, x_{2}\right),\left(x_{4}, x_{1}\right),\left(x_{4}, x_{2}\right),\left(x_{4}, x_{3}\right)\right\}$

Relation binaire correspondante (couple d'éléments de $E$ dont la concordance est supérieure ou égale à 4 et dont la discordance est inférieure ou égale à 1,6$)$

$$
\begin{aligned}
I_{(80 \%, 40 \%)}= & Q_{80 \%} \cap C D_{40 \%} \\
= & \left\{\left(x_{1}, x_{1}\right),\left(x_{2}, x_{2}\right),\left(x_{3}, x_{3}\right),\left(x_{4}, x_{4}\right),\right. \\
& \left.\left(x_{1}, x_{3}\right),\left(x_{1}, x_{4}\right),\left(x_{2}, x_{4}\right),\left(x_{3}, x_{4}\right)\right\}
\end{aligned}
$$

Représentons cet ensemble sous forme de graphe : deux sommets sont liés par une flèche orientée du premier vers le second si - et seulement si - le couple d'éléments de $E$ qu'il constitue appartient à $I_{80 \%, 40 \%}$

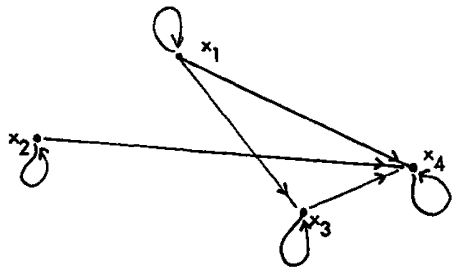

Figure 14

Cette relation binaire est transitive, antisymétrique et réflexive, c'est un ordre ${ }^{(1)}$ ) (Cet ordre n'est pas total puisque les éléments $x_{1}$ et $x_{2}$ sont incomparables.)

Il nous reste à le transformer en ordre total.

Pour cela nous faisons le calcul du rang moyen.

(1) Si elle n'avait pas été transitive, nous l'aurions complétée en prenant sa fermeture transitive. $\mathrm{n}^{\circ}$ janvier 1975, V-1. 
Calculons le rang $f$ pour les 4 éléments :

$$
f\left(x_{1}\right)=f\left(x_{2}\right)=1
$$

parce qu'aucun élément ne leur est préféré.

$$
f\left(x_{3}\right)=3
$$

parce que $x_{3}$ vient après $x_{1}$ dont le rang est 1 et qu'il y a deux éléments qui ont pour rang 1 .

$$
f\left(x_{4}\right)=4
$$

parce que $x_{4}$ vient après $x_{3}$ dont le rang est 3 et qui est le seul à avoir ce rang 3 .

Calculons le rang $g$ par une méthode analogue appliquée en sens inverse :

$$
g\left(x_{4}\right)=1
$$

parce qu'aucun élément ne lui est inférieur

$$
g\left(x_{3}\right)=g\left(x_{2}\right)=2
$$

parce que $x_{4}$, de rang 1 , est le seul élément qui leur soit inférieur.

$$
g\left(x_{1}\right)=4
$$

parce que $x_{3}$, de rang 2, est inférieur à $x_{1}$ et qu'il y a deux éléments qui ont pour rang 2 .

Le calcul du rang moyen $r$ découle de la formule :

$$
r(x)=\frac{n+1+f(x)-g(x)}{2}
$$

Nous aurons donc :

$$
\begin{aligned}
& r\left(x_{1}\right)=1 \\
& r\left(x_{2}\right)=2 \\
& r\left(x_{3}\right)=3 \\
& r\left(x_{4}\right)=4
\end{aligned}
$$

Notre classement final sera donc :

$$
x_{1}>x_{2}>x_{3}>x_{4}
$$

\subsection{Conclusion et exemples d'utilisation}

Ainsi, à partir d'un $m$-uplet de préordres pondérés quelconques sur $E$, on obtient un préordre total sur $E$. Dans la méthode Electre classique, chaque point de vue devait correspondre non pas seulement à un préordre quelconque, mais à une série de notes; tandis que le résultat était seulement la donnée d'un groupe de 1 ou plusieurs éléments non surclassés (le noyau).

Notre méthode présente ainsi une économie d'information initiale, et un supplément d'information final. 
En voici un résumé graphique :

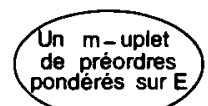

calcul des rangs moyens d'où l'on déduit $Q_{k}, D_{l}$, puis la relation linéaire :

$$
I_{k, l}=Q_{k} \cap C D_{l}
$$

ne partie

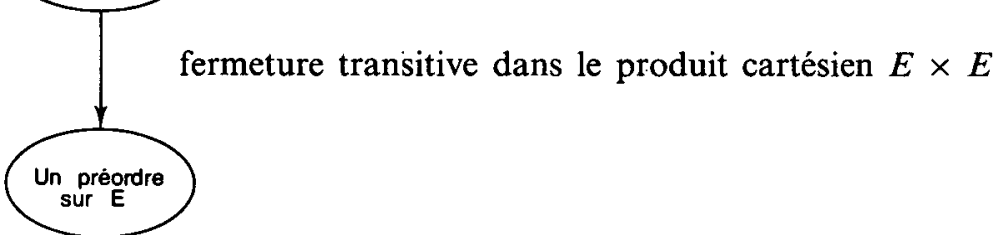

rangement suivant le rang moyen

Figure 15

\section{REMARQue :} étapes :

Il est à noter que cette transformation d'Electre s'est faite en plusieurs

- en 1970, nous en avons fait une $1^{\mathrm{e}}$ modification dans le cadre du Cetem (voir [3], [7]) en développant en particulier les rangs $f$ et $g$.

- En 1971, la SEMA créait Electre II qui à partir de données ordinales ou cardinales aboutissait à un préordre total (voir [6], [7]).

Nous avons fait des essais sur des ensembles de faible dimension, à partir de préordres totaux, dans deux domaines très différents :

a) Dans le domaine maritime :

Un groupe d'experts en matière de navigation avaient classés un ensemble de 23 caboteurs, suivant les 10 points de vue que voici :

recettes

soutes

frais de port

forme

longueur des écoutilles (aptitude à la charge de conteneurs)

largeur des écoutilles (idem)

portée

tirant d'eau

aides à la navigation (présence éventuelle de goniomètres, etc...)

nombres de ponts (les « sheltered-dock » étant assimilés aux 2 ponts).

$\mathrm{n}^{\circ}$ janvier 1975, V-1. 
Chaque point de vue donnait un préordre total sur l'ensemble $E$ des 23 caboteurs.

Ces préordres étaient pondérés suivant des considérations de rentabilité.

Il s'agissait, à partir de ces 10 points de vue différents, d'obtenir un classement complet sur notre ensemble de 23 caboteurs, et de pouvoir dire ainsi lesquels étaient meilleurs que les autres.

L'application de la dérivée d'Electre a donné de bons résultats $\left({ }^{1}\right)$.

b) En sociologie

Lors d'une étude comparative sur les emplois du temps dans les divers pays du monde, suivant les critères sexe et état matrimonial, on s'est amusé à considérer (suivant l'optique d'un individu parfaitement paresseux et bon vivant) qu'un emploi du temps était meilleur qu'un autre s'il correspondait à une durée quotidienne plus faible en travail, en transport obligatoire, et en ménage; et à une durée plus longue au contraire en repos, en sommeil, en télévision et en autres loisirs divers (certaines rubriques étaient considérées a priori comme neutres, n'apportant ni de l'agrément, ni du désagrément, pour un individu paresseux et bon vivant : tel était le cas pour les courses, la toilette et le soin aux enfants).

A partir de ces préordres totaux sur l'ensemble $E$ des catégories humaines considérées, notre programme a montré, par exemple, que l'emploi du temps le plus enviable de tous était celui des femmes mariées des pays d'Europe de l'ouest!

REMARQUE : Le programme, testé dans le cas où les données de base sont des préordres totaux, ne semble pas poser de difficultés particulières.

\section{ANNEXE \\ RAPPEL DE CERTAINES DEFINITIONS MATHEMATIQUES}

Soit $E$ un ensemble fini quelconque.

Préordre :

Un préordre $P$ sur $E$ est une relation binaire réflexive et transitive.

\section{Préordre total :}

Un préordre $P$ sur $E$ est dit " total ", s'il permet de comparer tout couple d'éléments de $E:(\forall x \in E)(\forall y \in E)(x P y$ ou $y P x)$.

(1) Notons que cette même méthode a été également appliquée pour étudier l'approfondissement optimal du chenal de Douala. 
Ordre (1):

Un ordre $R$ sur $E$ est une relation binaire réflexive, antisymétrique et transitive.

Ordre total :

Un ordre $R$ sur $E$ est dit «total» s'il permet de comparer tout couple d'éléments de $E$.

\section{Relation d'équivallence :}

Une relation d'équivallence sur $E$ est une relation binaire réflexive, symétrique et transitive.

\section{Treillis :}

Un treillis est un ensemble ordonné tel que chaque couple $(x, y)$, d'éléments admette un surpremum et un infimum.

\section{Majorant-minorant :}

Soit $R$ une relation d'ordre sur $E$.

Soient $x$ et $y$ deux éléments quelconques différents de $E$.

On dit que " $x$ est un majorant de $y$ » si et seulement si $x R y$.

De même, on dit que " $x$ est un minorant de $y$ » si et seulement $y R x$.

\section{Majorant-universel :}

Soit $R$ une relation d'ordre sur $E$.

Soit $x$ un élément de $E$.

On dit que « $x$ est un majorant universel de $E »$ si et seulement si on a : $(\forall y \in E)(x R y)$.

\section{Intervalle-intermédiaire :}

Si $x$ minore $y$, on appelle intervalle $[x, y]$, l'ensemble des éléments intermédiaires entre $x$ et $y$, c'est-à-dire l'ensemble des éléments $t$ tels que : $x R t$ et $t R y$.

\section{Prédécesseur-Successeur :}

On dit que $x$ succède à $y$ si et seulement si $x$ est un majorant de $y$, distinct de $y$, et tel qu'il n'y ait aucun élément intermédiaire entre $x$ et $y$.

(1) Ou « ordre au sens large ", diffère de l'ordre strict par sa réflexivité. 
Maximal-Minimal :

Soit $X$ une partie d'une ensemble ordonné $E$.

Soit $x$ un élément de $E$.

On dit que " $x$ est maximal », si et seulement si : (i) $x \in X$

Maximum-Minimum :

(ii) $\left(\exists / y^{\prime} \neq x\right)(y R x$ et $y \in X)$

Soit $X$ une partie d'un ensemble ordonné $E$.

Soit $x$ un élément de $E$.

On dit que " $x$ est maximal ", si et seulement si : (i) $x \in X$

(ii) $(\forall y \in X)(x R y)$

Sup. demi treillis :

Ordre tel que chaque couple d'éléments de $E$ admette un plus petit majorant commun.

Inf. demi treillis :

Ordre tel que chaque couple d'éléments de $E$ admette un plus grand minorant commun.

\section{Chaine :}

Sous-ensemble totalement ordonné de $E$.

Longueur d'une chaîne:

Soit $m$ le nombre d'élément d'une chaîne $C$.

On dit que la longueur de cette chaîne est $m-1$.

Longueur d'un ensemble ordonné :

Par définition, c'est le supremum de la longueur de ses chaînes c'est-à-dire le plus petit des majorants de ses longueurs.

\section{Hauteur d'un élément :}

Soit $E$ un ensemble ordonné de longueur finie, ayant un minorant universel $O$.

On appelle hauteur d'un élément $x$ de $E$, la longueur de l'intervalle $[O, x]$ : $h(O)=l([O, x])$.

Condition de Jordan Dedekind :

Toutes les chaînes maximales entre 2 éléments ont la même longueur. 
Un ensemble $E$ ordonné par $R$ est semi-modulaire supérieurement s'il vérifie la condition suivante :

Si $x$ et $y$ succèdent à un même élément $z$, ils précèdent un même quatrième $t$.

C'est la condition du quadrilatère.

Profondeur d'un élément :

Soit $E$ un ensemble ordonné, de longueur finie, admettant un majorant universel $u$.

On appelle "profondeur " d'un élément $x$ de $E$, la longueur de l'intervalle $[x, u]$ :

$$
p(x)=l([x, u])
$$

\section{Dimension-Codimension :}

Soit $E$ un ensemble ordonné admettant un minorant universel $O$ (dualement « majorant universel » $u$ ).

On appelle dimension (dualement "codimension") l'application $d$ suivante :

$$
\begin{aligned}
d(x) & =h(x)-1 \\
(\operatorname{cod}(x) & =p(x)-1)
\end{aligned}
$$

\section{Théorème :}

Un ensemble ordonné (de longueur finie) semi-modulaire supérieurement, vérifie la condition de Jordan Dedekind.

" Graduation " ou "Fonction de rang "

Un ensemble ordonné $E$ est gradué s'il existe une application $g$ de $E$ dans l'ensemble des entiers positifs ou négatifs $Z$, vérifiant :

$$
\begin{aligned}
& \text { i) } \quad(x R y \text { et } y \not R x) \\
& \text { ii) } \quad x \text { précède } y \Rightarrow g(y) \leqslant g(x)) \\
& \Rightarrow g(x)+1
\end{aligned}
$$

L'application $g$ est appelée "graduation ", ou "fonction de rang".

Théorème : Un ensemble ordonné gradué (de longueur finie) vérifie la condition de Jordan Dedekind.

Théorème : Un ensemble ordonné, avec minorant (ou majorant) universel vérifie la condition de Jordan Dedekind si et seulement si il est gradué. 
En conclusion : Appelons $\Omega$ l'ensemble des ensembles ordonnés de longueur finie.

Soit $S M$ le sous-ensemble des semi-modulaires.

Soit $G$ le sous-ensemble des gradués.

Soit $J D$ le sous-ensemble de ceux qui vérifient la condition de Jordan Dedekind.

Soit $M U$ le sous-ensemble de ceux qui admettent un minorant universel.

Nous avons :

$$
\begin{aligned}
S M & \subset J D \\
G & \subset J D \\
S M \cap M U & \subset G \cap M U \\
G \cap M U & =M U \cap J D
\end{aligned}
$$

D'où le graphique :

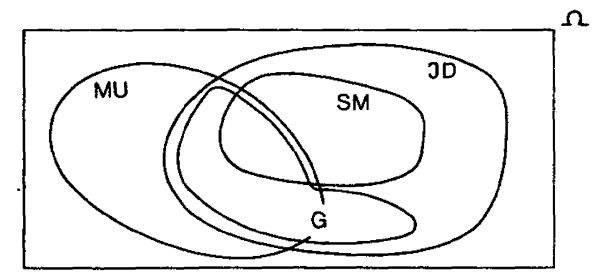

Figure 16

\section{BIBLIOGRAPHIE}

[1] BaRbut M. et MONJARET B., Ordre et classification, algèbre et combinatoire, UPI 1972 (tomes 1 et 2).

[2] Benayoun R., Roy B., Sussmann B., Manuel de référence du programme Electre, Note de synthèse, Formation, $\mathrm{n}^{\circ} 25$. Direction scientifique Sema, juin 1966.

[3] BERnARD G. et BESSON M., Douze méthodes d'analyse multicritère, RIRO, $\mathrm{n}^{\circ} \mathrm{V}-3,1971$.

[4] Besson M., Iphi, ou un nouveau procédé de typologie. Séminaires de l'IRIA, classification automatique et perception par ordinateur, 1973.

[5] Besson M., A propos des distances entre ensembles de parties, Mathématiques et sciences humaines, 11 année, $\mathrm{n}^{\circ} 42,1973$.

[6] Crolleau, et Tergny J., Manuel de référence du prog. Electr. II, déc. 1971.

[7] Guigou J. L., Analyse des données et choix à critères multiples, Dunod, 1973.

[8] LERMAN I. C., Les bases de la classification automatique, Gauthier Villars, 1970.

[9] RoY B., Algèbre moderne et théorie des graphes, Dunod, 1969.

[10] Roux M., "Algorithmes pour construire une hiérarchie particulière ». Thèse de $3^{e}$ cycle. Stat. math. Déc. 1968 . UNIV, Paris.

Revue Française d'Automatique, Informatique et Recherche Opérationnelle $n^{\circ}$ janv. 1975, V-1. 